\title{
Percepción en la planificación de cursos de física: relaciones en entornos sincrónicos
} Perception in the planning in physics courses: relationship in synchronous environments

\author{
Jorge Fernando Márquez Peñaranda ${ }^{1}$, \\ Mawency Vergel Ortega ${ }^{2}$, Henry de Jesús \\ Gallardo Pérez ${ }^{3}$ \\ Universidad Francisco de Paula Santander
}

\section{RESUMEN}

La investigación se realizó con estudiantes de física I y II de universidades del Departamento Norte de Santander-Colombia. Su objetivo fue establecer la relación entre la planificación de las clases de los profesores y las percepciones de los estudiantes en entornos sincrónicos. Cuatro instrumentos midieron la percepción de los estudiantes respecto a la práctica pedagógica de los profesores de física. La investigación siguió un enfoque cuantitativo correlacional.

1 Ingeniero civil. PhD en ingeniería. Universidad Francisco de Paula Santander. Correo. jorgefernandomp@ ufps.edu.co. Orcid: https://orcid.org/0000-0002-9193-8349

2 Licenciada en Matemáticas y Física PhD en Educación. Magister en Gerencia educativa. Universidad Francisco de Paula Santander. Correo. mawencyvergel@ufps. edu.co. Orcid: https://orcid.org/0000-0001-8285-2968

3 Licenciado en matemáticas y Física. PhD en Educación. Magister en Economía. Correo. henrygallardo@ ufps.edu.co. Orcid: https://orcid.org/0000-0003-4377-3903
Resultados de la investigación muestran que la percepción de los estudiantes sobre la planeación de clase no guarda una asociación estrecha respecto a la consideración que tienen los profesores, encuentros vía streaming muestra asociación a percepción de enfoque, y resultados de aprendizaje. Se establece correlación alta entre la implementación de consideraciones contempladas en el enfoque dialógico-crítico y por competencias, por parte de un alto porcentaje de profesores. La contribución de las acciones realizadas por los docentes para potenciar el desarrollo del pensamiento variacional de los estudiantes a través del quehacer académico y pedagógico, tiene una relación alta entre lo que el profesor realiza y resultados de aprendizaje obtenidos por los estudiantes. 
PALABRAS CLAVE: Educación superior, planeación, pensamiento, correlación, resultados de aprendizaje, sincrónico.

\section{ABSTRACT}

The research was carried out with physics I students from universities in the North Department of Santander-Colombia. Its objective was to establish the relationship between teachers' lesson planning and students' perceptions in synchronous environments. Four instruments measured the students' perception of the pedagogical practice of physics teachers. The research followed a quantitative correlational approach. Research results show that, students' perception of lesson planning does not keep a close association with teachers' consideration, streaming encounters show association with perception of focus, and learning outcomes. A high correlation is established between the implementation of considerations contemplated in the dialogic-critical approach and by competencies, on the part of a high percentage of teachers. The contribution of the actions carried out by the teachers to enhance the development of students' variational thinking through academic and pedagogical work, has a high relationship between what the teacher does and the learning results obtained by the students.

KEYWORDS: Higher education, planning, thinking, correlation, learning outcomes, synchronic.

\section{INTRODUCCIÓN}

La física es considerada la base de procesos complejos de conocimiento, donde es necesario el pensamiento crítico, reflexivo y analítico; ésta, desarrolla la capacidad para crear, razonar, formular y solucionar problemas, cobrando importancia en los primeros pasos de la formación intelectual de las personas en los procesos de abstracción. Sin embargo, en
Norte de Santander, se presentan dificultades en su aprendizaje, constituyéndose en un área de alta repitencia y causal de deserción en los primeros semestres [1]. Lo considerado entonces importante es establecer propósitos y funciones decididos en cada institución y cada programa para adelantar cada clase. En el ámbito internacional, para Borrero, el éxito se asocia al planificar, ejecutar de acuerdo a metas y propósitos proyectados y emitir un juicio evaluativo sobre sus realizaciones [2]. La Universidad en Norte de Santander además se encuentra inmersa en la falta de profesionales formados en esta ciencia, la mayoría de cursos son orientados por ingenieros, y sus orientaciones en algunos casos, aún se asocian a transmisión verbal de contenidos[3], la relación profesor estudiante tiene una baja interacción y poco trabajo mancomunado, predominando sujetos reproductores del discurso, orientado a solucionar problemas de libros [2], sin que desarrolle habilidades de la vida cotidiana y adquirir competencias que eviten el manejo de conceptos, los cuales generan respuestas inadecuadas o incluso incorrectas [4]. Aunque se realizan paralelos cursos de laboratorio, resultados de pruebas saber muestran puntajes por debajo del promedio en las ingenierías [5].

En las universidades de la región Nortesantandereana, en Colombia, prácticas de planificación se fundamenta según el Proyecto Educativo Institucional, según currículo y acorde a cada programa sigue un micro currículo, así mismo algunas ya implementan diseños de formatos en los cuales incorporan resultados de aprendizaje, competencias, objetivos de aprendizaje acorde apolíticas nacionales, como proceso de planeación[6], lo cual se ha fortalecido en tiempos de pandemia al ser productivo para procesos de asesoría y clases a través de medios sincrónicos [7]. La planeación, no obstante, se aproxima a elementos contextualizados acorde a la producción colaborativa por parte de estudiantes y profesores mediante el 
acercamiento de los objetos de estudio con el entorno [8]. La investigación analiza el aporte que los modelos pedagógicos manifiestos en los proyectos educativos de las universidades hacen mediante la planeación de estrategias pedagógicas como herramientas mediadoras [9] para el aprendizaje en el contexto de la educación física.

El objetivo que abordó esta investigación fue Correlacionar la planificación de estrategias para la enseñanza de las fisica manifiesta por los docentes con la percepción que tienen los estudiantes sobre el proceso planificación en medio sincrónico por parte de los estudiantes de Fìsica I de diferentes programas acadèmicos de Universidades del Departamento Norte De Santander-Colombia.

\section{MÉTODO}

La investigación se aborda desde el paradigma cuantitativo [10] con apoyo cualitativo [11], sigue un método correlacional y utiliza método deductivo. La hipótesis central que se planteó en esta investigación fue: La planificación en la enseñanza de las fisica se relaciona con percepción de estudiantes. La población objeto está constituida por 1500 estudiantes que cursan física I o Física II en Universidades del departamento Norte de Santander (Francisco de Paula Santander UFPS, Simón Bolívar, Corporación universitaria Minuto de Dios Uniminuto, Universidad Libre, Universidad Nacional Abierta y a distancia). Realizado muestreo por conglomerados, la muestra estuvo constituida por tres (3) conglomerados; (200) estudiantes y diez (5) profesores de la UFPS, noventa y cinco (50) estudiantes, y dos (2) profesores de la Universidad abierta y a distancia, cincuenta (50) estudiantes y dos (2) profesores de la Simón Bolívar. La investigación siguió fases de exploración [12], diseño, validación de Instrumentos, con encuesta escala tipo likert utilizada para caracterizar las estrategias pedagógicas empleadas al planificar sus procesos de enseñanza para orientar el programa. Así mismo se diseñó test prueba de ejercicios y resolución de problemas y test de aplicación utilizando la métrica fuzzy [13], con el propósito de medir pensamiento variacional [14]. Resultados que apoyan en portafolios, documentosque contienenaspectos curriculares, cartillas y material de las instituciones. En la tabla 1 , se muestra la dimensión, indicador e ítem de las preguntas elementos, que fueron necesarios para la operacionalización de las variables en esta investigación. Para determinar la fiabilidad de los instrumentos aplicados, se calculó el estadístico de fiabilidad Alfa de Cronbach: el instrumento 1 (17 ítems, aplicado a profesores, tiene como objetivo caracterizar la planificación educativa del docente), con . El instrumento 2 [15] (17 ítems, la respuesta para cada ítem corresponde con la realidad pedagógica y académica percibida por los estudiantes), con , validez a través de juicio de expertos Kappa>0.7. Establecida la correlación entre instrumentos 1 y 2 aplicados a profesores y estudiantes respectivamente acorde a planteamientos de Isidro, Vergel, Martínez [16], se determinan probabilidades conjuntas por técnicas de correlación, Gamma, Tau de Kendal y medida de acuerdos mediante índice Kappa de Cohen [17].

\section{ANÁLISIS, INTERPRETACIÓN Y DISCUSIÓN DE RESULTADOS}

Análisis documental y cualitativo frente a prácticas utilizadas por instituciones educativas se obtienen categorías emergentes de análisis de preconcepciones, actividades preliminares, actividades durante y finalizado el programa curricular, control y registro de actividades, evaluación, manejo del tiempo, créditos, construcción de códigos y prototipos ( Tabla 1), con base en ello se operacionalizan variables para ajustar el diseño de test. 
Tabla 1. Operacionalización variable planificación práctica pedagógica

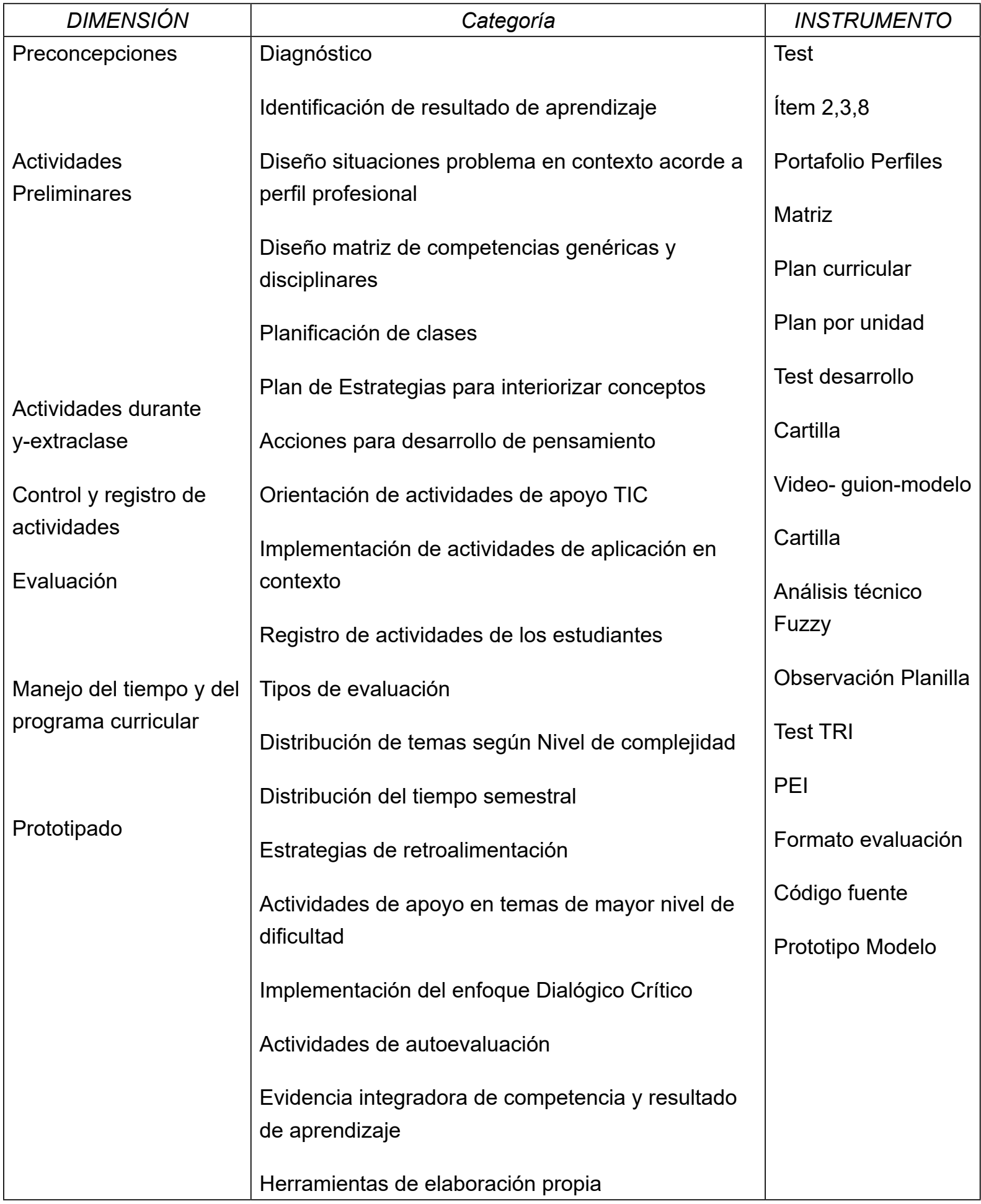


Los resultados de los test, muestran las probabilidades conjuntas entre los ítems del instrumento 1 (encuesta aplicada a los profesores), con los ítems correspondientes del instrumento 2 (encuesta aplicada a los estudiantes), revelan en general que, la percepción de los estudiantes con respecto a la práctica de los docentes concuerda en la implementación de enfoque, uso herramientas TIC y estrategias de evaluación integradora, sin embargo, no existe acuerdo en la planeación de la clase.

Tabla 2. Cruce de ítems 1 a 5. Porcentaje de respuestas conjuntas estudiantes y profesores

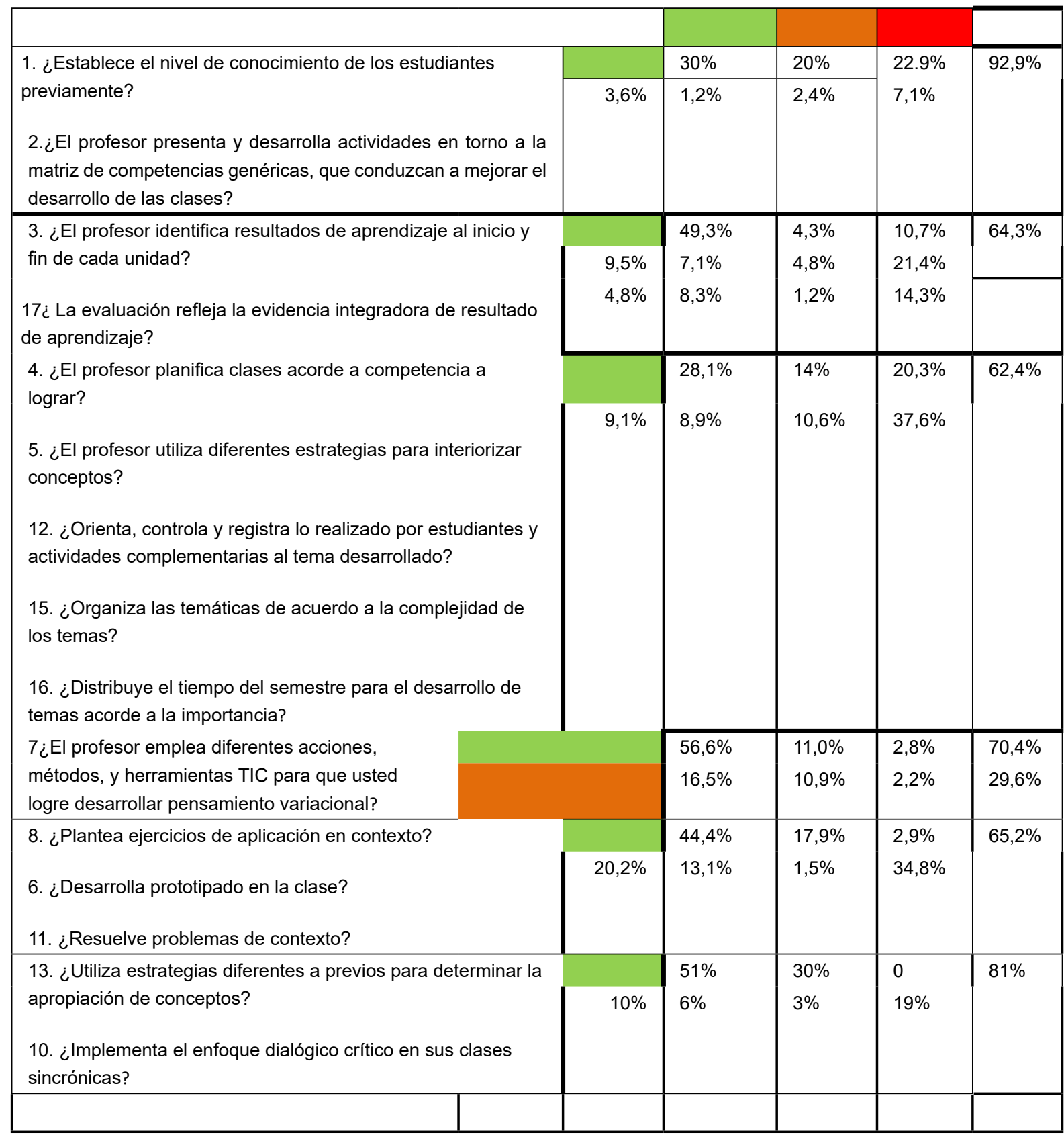


Respecto a la correlación de ítems, $p>0.05$, indicando que la intensidad de la relación entre lo que manifiestan los profesores que realizan y lo que perciben los estudiantes en ítems asociados a planeación no es significativa, también, el índice Kappa de Cohen con valor $p>0.05$ muestra acuerdos no significativos entre estudiantes y profesores para la misma pregunta. Sin embargo, en ítems asociados a resultado de aprendizaje, evaluación y enfoque, el profesor implementa el enfoque crítico dialógico en la realización de las clases, su probabilidad conjunta no presenta diferencias significativas, las proporciones para cada cruce de categorías son similares, por lo cual existe equilibrio entre lo que manifiestan realizan los profesores y lo que perciben los estudiantes. Frente a si la evaluación refleja la evidencia asociada a resultado de aprendizaje, existe acuerdo $p=$ 0.05 , sin embargo, al preguntar por concepción de resultado de aprendizaje, este se centra es en objetivo o procedimientos. La contribución de las acciones realizadas y estrategias aplicadas por los docentes en la adquisición del pensamiento variacional de los estudiantes a través del quehacer académico y pedagógico, tiene una relación alta entre lo que el profesor realiza y los resultados de aprendizaje obtenidos por los estudiantes. Frente a test 3 , estudiantes manifiestan que existen diferentes estrategias y herramientas utilizadas por profesores, aunque señalan que algunos profesores no utilizan estrategias (limitadas a lectura de presentaciones), las actividades realizadas por el docente para mejorar la comprensión de los temas en los cuales presentan mayor dificultad los estudiantes, está asociada con percepción del estudiante hacia el docente, así como un mal manejo del tiempo por unidad de aprendizaje y la sobrecarga de tareas extracurriculares o no uso de herramientas interactivas o ausencia en encuentros vía stremming, se asocian a baja calidad. La plataforma utilizada no se socia a la calidad de percepción del profesor, pero si a planeación y evaluación.

Tabla 2. Asociación entre ítems profesor versus estudiantes

\begin{tabular}{|c|c|c|c|c|c|}
\hline \multicolumn{6}{|c|}{ Medidas simétricas } \\
\hline \multicolumn{2}{|c|}{$8,10,11,13,6,3$} & & Error típ. & & Sig. \\
\hline Ordinal por & Tau-b de Kendall & ,072 & ,117 & ,358 & ,700 \\
\hline & Tau-c de Kendall & ,034 & ,068 & ,368 & ,700 \\
\hline & Gamma & ,150 & ,395 & ,358 & ,700 \\
\hline $\begin{array}{l}\text { Medida de } \\
\text { acuerdo }\end{array}$ & Kappa &,- 022 & ,048 &,- 492 & ,600 \\
\hline & & & & & \\
\hline
\end{tabular}


Análisis documental acorde a material requerido por universidades para soporte de clases sincrónicas, ya sean formatos o inclusión de actividades en plataformas de asistencia se observa que el $72 \%$ de los profesores si realizan planificación detallada de su clase y ajustan acorde a perfiles, no obstante, esto no es percibido por los estudiantes según análisis de test.

Existe relación significativa entre la implementación del enfoque pedagógico, su implementación en la realización de las clases de física I, no obstante, un $40 \%$ señaló centrarse en aplicación a problemas de física de libros de textos como situaciones de contexto, y un $47 \%$ manifestó que utilizan sólo videos de apoyo de la web para dar a conocer experimentos. Al igual que lo señala Isidro, Vergel y Martínez [16] en Cálculo, percepciones de estudiantes difieren de lo manifiesto por docentes.

\section{CONCLUSIONES}

La percepción de los estudiantes frente a la práctica pedagógica de los docentes en física en medio sincrónico, no es acorde a las actividades y estrategias que los profesores manifiestan realizar.

Existe relación significativa entre la implementación del enfoque pedagógico, su implementación en la realización de las clases de física I por parte de los docentes y la apreciación de los estudiantes, altamente asociada a resultados de aprendizaje de los estudiantes.

Inadecuado uso del tiempo por unidad de aprendizaje, sobrecarga de trabajos extracurriculares, no uso de herramientas interactivas o ausencia en encuentros vía stremming se asocian a baja percepción en la calidad en la formación por parte del profesor.

La contribución de las acciones realizadas y estrategias aplicadas por los docentes en la adquisición del pensamiento variacional de los estudiantes a través del quehacer académico y pedagógico, tiene una relación alta entre lo que el profesor realiza y los resultados de aprendizaje obtenidos por los estudiantes.

\section{REFERENCIAS BIBLIOGRÁFICAS}

[1] Rodríguez, A., Jiménez Y., Herrera L. y Carrera O., 2019. Evaluación de estrategias que impulsan en el ciudadano el uso de las plataformas de e-gobierno municipal: un enfoque de dinámica de sistemas Reci 815 DOI: 10.23913/reci.v8i15.91

[2] Nieto Contreras J F, Rojas-Suarez J P y Vergel- Ortega M 2019 Impacto de estrategia pedagógica basada en el aprendizaje creativo para estudiantes de ingeniería (Bogotá: Ecoe Ediciones)

[3] Gómez CS, Rojas JP y Vergel M 2020 Estrategia gerencial seis sigma en la gestión de la vicerrectoría de bienestar universitario. (Bogotá: ECOE ediciones)

[4] Karademir E and Ulucinar U 2017 Examining the Relationship between Middle School Students' Critical Reading Skills, Science Literacy Skills and Attitudes: A Structural Equation Modeling Journal of Education in Science, Environment, and HealthBrue 1215

[5] Cardoso, E. O. \& Cerecedo, M. T. (2008). El desarrollo de las competencias física en la primera infancia. Revista iberoamericana de educación, n 47

[6] Mendez-Molina B L, Moreno L y VergelOrtega M 2019 Infección de aislados nativos de Burkholderia glumae en variedades de semillas de arroz certificadas (Bogotá: ECOE Ediciones) 
[7] Maldonado $\mathrm{H}$, Vergel-Ortega, $\mathrm{M}$ y Rojas-Suárez J P 2020 Innovación y creatividad: una estrategia pedagógica en la enseñabilidad del cálculo aplicado a la física electromagnética (Bogotá: Ecoe ediciones)

[8] Borrero, A. (2008). La universidad. Estudios sobre sus orígenes, dinámicas y tendencias, vol 1 . Historia universitaria: La universidad en Europa desde sus orígenes hasta la Revolución Francesa, Bogotá: Universidad Javeriana

[9] Romero E, Torres JM y Vergel M 2020 Representaciones sociales de estudiantes universitarios sobre el aprendizaje del cálculo y la química (Bogotá: ECOE Ediciones)

[10] Carrero F 2017 La comprensión lectora en el alumnado sordo desde la perspectiva de la escuela inclusiva IJERI: International Journal of Educational Research and Innovation 8206

[11] Ángulo L. y León, A. (2005). Perspectiva crítica de Paulo Freire y su contribución a la teoría del currículo. Educare, 9 (29), pp. 159-164

[12] Carr, W. \& Kemmis, S. (1988). Teoría crítica de la enseñanza. Barcelona: Martínez Roca.

[13] Peláez M 2013 Dimensions of rules and their correspondence to rule-governed behavior European Journal of Behavior Analysis 14259

[14] Hoffman S and Lewis J 2001 Square functions of Calderon type and application Rev Mat Iberoam 634

[15] Rusilowati A, Kurniawati L, Nugroho S and Widiyatmoko A2016 Developing an Instrument of Scientific Literacy
Assessment on the Cycle Theme. International Journal of Environmental \& Science Education 11 5718-5727

[16] Isidro H, Martínez Lozano J y Vergel M 2016 Desarrollo del pensamiento matemático en estudiantes de cálculo integral su relación con la planificación docente. Revista Científica, 23(3), 1729.

[17] Ernest, P. (2004). "What is the Philosophy of Mathematics Education?", Philosophy of Mathematics and Education Journal 18 